\title{
Resistance to Empiric Antimicrobial Treatment Predicts Outcome in Severe Sepsis Associated with Gram-Negative Bacteremia
}

\author{
Scott T. Micek, PharmD1, Emily C. Welch, PharmD¹, Junaid Khan, MD², Mubashir Pervez, MD², Joshua A. Doherty, BA³, \\ Richard M. Reichley, RPh ${ }^{3}$, Joan Hoppe-Bauer, BA, BS, MT4, W. Michael Dunne, $\mathrm{PhD}^{4}$, Marin H. Kollef, MD²*
}

\begin{abstract}
${ }^{1}$ Pharmacy Department, Barnes-Jewish Hospital, St. Louis, Missouri; ${ }^{2}$ Pulmonary and Critical Care Division, Washington University School of Medicine, St. Louis, Missouri; ${ }^{3}$ Hospital Informatics Group, BJC Healthcare, St. Louis, Missouri; ${ }^{4}$ Laboratory Medicine Department, Barnes-Jewish Hospital, St. Louis, Missouri.
\end{abstract}

BACKGROUND: Gram-negative bacteria are an important cause of severe sepsis. Recent studies have demonstrated reduced susceptibility of Gram-negative bacteria to currently available antimicrobial agents.

METHODS: We performed a retrospective cohort study of patients with severe sepsis who were bacteremic with Pseudomonas aeruginosa, Acinetobacter species, or Enterobacteriaceae from 2002 to 2007. Patients were identified by the hospital informatics database and pertinent clinical data (demographics, baseline severity of illness, source of bacteremia, and therapy) were retrieved from electronic medical records. All patients were treated with antimicrobial agents within 12 hours of having blood cultures drawn that were subsequently positive for bacterial pathogens. The primary outcome was hospital mortality.

RESULTS: A total of 535 patients with severe sepsis and Gram-negative bacteremia were identified. Hospital mortality was $43.6 \%$, and $82(15.3 \%)$ patients were treated with an antimicrobial regimen to which the causative pathogen was resistant. Patients infected with a resistant pathogen had significantly greater risk of hospital mortality (63.4\% vs $40.0 \% ; P<0.001)$. In a multivariate analysis, infection with a pathogen that was resistant to the empiric antibiotic regimen, increasing APACHE II scores, infection with Pseudomonas aeruginosa, healthcare-associated hospital-onset infection, mechanical ventilation, and use of vasopressors were independently associated with hospital mortality.

CONCLUSIONS: In severe sepsis attributed to Gramnegative bacteremia, initial treatment with an antibiotic regimen to which the causative pathogen is resistant was associated with increased hospital mortality. This finding suggests that rapid determination of bacterial susceptibility could influence treatment choices in patients with severe sepsis potentially improving their clinical outcomes. Journal of Hospital Medicine 2011;6:405-410. (c) 2011 Society of Hospital Medicine
Severe sepsis and septic shock are associated with excess mortality when inappropriate initial antimicrobial therapy, defined as an antimicrobial regimen that lacks in vitro activity against the isolated organism(s) responsible for the infection, is administered. ${ }^{1-4}$ Unfortunately, bacterial resistance to antibiotics is increasing and creates a therapeutic challenge for clinicians when treating patients with serious infections, such as severe sepsis. Increasing rates of bacterial resistance leads many clinicians to empirically treat critically ill patients with broad-spectrum antibiotics, which can perpetuate the cycle of increasing resistance. ${ }^{5,6}$ Conversely, inappropriate initial antimicrobial therapy can lead to treatment failures and adverse patient outcomes. ${ }^{7}$ Individuals with severe sepsis

*Address for correspondence and reprint requests: Marin H. Kollef, MD, Division of Pulmonary and Critical Care Medicine, Washington University School of Medicine, 660 South Euclid Avenue, Campus Box 8052, St. Louis, MO 63110; Tel.: 314-454-8764;

E-mail: mkollef@dom.wustl.edu

Additional Supporting Information may be found in the online version of this article.

Received: June 15, 2010; Revised: October 21, 2010; December 13, 2010; Accepted: December 15, 2010

2011 Society of Hospital Medicine DOI 10.1002/jhm.899

Published online in Wiley Online Library (Wileyonlinelibrary.com). appear to be at particularly high risk of excess mortality when inappropriate initial antimicrobial therapy is administered. ${ }^{8,9}$

The most recent Surviving Sepsis Guidelines recommend empiric combination therapy targeting Gramnegative bacteria, particularly for patients with known or suspected Pseudomonas infections, as a means to decrease the likelihood of administering inappropriate initial antimicrobial therapy. ${ }^{10}$ However, the selection of an antimicrobial regimen that is active against the causative pathogen(s) is problematic, as the treating physician usually does not know the susceptibilities of the pathogen(s) for the selected empiric antibiotics. Therefore, we performed a study with the main goal of determining whether resistance to the initially prescribed antimicrobial regimen was associated with clinical outcome in patients with severe sepsis attributed to Gram-negative bacteremia.

\section{MATERIALS AND METHODS Study Location and Patients}

This study was conducted at a university-affiliated, urban teaching hospital: Barnes-Jewish Hospital (1200 beds). During a 6-year period (January 2002 to December 2007), all hospitalized patients with a positive blood culture for Gram-negative bacteria, with 
antimicrobial susceptibility testing performed for the blood isolate(s), were eligible for this investigation. This study was approved by the Washington University School of Medicine Human Studies Committee.

\section{Study Design and Data Collection}

A retrospective cohort study design was employed. Two investigators (J.A.D., R.M.R.) identified potential study patients by the presence of a positive blood culture for Pseudomonas aeruginosa, Acinetobacter species, or Enterobacteriaceae (Escherichia coli, Klebsiella species, Enterobacter species) combined with primary or secondary International Classification of Diseases (ICD-9-CM) codes indicative of acute organ dysfunction, at least two criteria from the systemic inflammatory response syndrome (SIRS), ${ }^{10}$ and initial antibiotic treatment with either cefepime, piperacillintazobactam, or a carbapenem (imipenem or meropenem). These antimicrobials represent the primary agents employed for the treatment of Gram-negative infections at Barnes-Jewish Hospital during the study period, and had to be administered within 12 hours of having the subsequently positive blood cultures drawn. Based on the initial study database construction, 3 investigators (E.C.W., J.K., M.P.) merged patient-specific data from the automated hospital medical records, microbiology database, and pharmacy database of Barnes-Jewish Hospital to complete the clinical database under the auspices of the definitions described below.

The baseline characteristics collected by the study investigators included: age, gender, race, the presence of congestive heart failure, chronic obstructive pulmonary disease, diabetes mellitus, chronic liver disease, underlying malignancy, and end-stage renal disease requiring renal replacement therapy. All cause hospital mortality was evaluated as the primary outcome variable. Secondary outcomes included acquired organ dysfunction and hospital length of stay. The Acute Physiology and Chronic Health Evaluation (APACHE) $\mathrm{II}^{11}$ and Charlson co-morbidity scores were also calculated during the 24 hours after the positive blood cultures were drawn. This was done because we included patients with community-acquired infections who only had clinical data available after blood cultures were drawn.

\section{Definitions}

All definitions were selected prospectively as part of the original study design. Cases of Gram-negative bacteremia were classified into mutually exclusive groups comprised of either community-acquired or healthcare-associated infection. Patients with healthcare-associated bacteremia were categorized as community-onset or hospital-onset, as previously described. ${ }^{12}$ In brief, patients with healthcare-associated community-onset bacteremia had the positive culture obtained within the first 48 hours of hospital admission in combination with one or more of the following risk factors: (1) residence in a nursing home, rehabilitation hospital, or other long-term nursing facility; (2) previous hospitalization within the immediately preceding 12 months; (3) receiving outpatient hemodialysis, peritoneal dialysis, wound care, or infusion therapy necessitating regular visits to a hospital-based clinic; and (4) having an immune-compromised state. Patients were classified as having healthcare-associated hospital-onset bacteremia when the culture was obtained 48 hours or more after admission. Community-acquired bacteremia occurred in patients without healthcare risk factors and a positive blood culture within the first 48 hours of admission. Prior antibiotic exposure was defined as having occurred within the previous 30 days from the onset of severe sepsis.

To be included in the analysis, patients had to meet criteria for severe sepsis based on discharge ICD-9CM codes for acute organ dysfunction, as previously described. ${ }^{13}$ The organs of interest included the heart, lungs, kidneys, bone marrow (hematologic), brain, and liver. Patients were classified as having septic shock if vasopressors (norepinephrine, dopamine, epinephrine, phenylephrine, or vasopressin) were initiated within 24 hours of the blood culture collection date and time. Empiric antimicrobial treatment was classified as being appropriate if the initially prescribed antibiotic regimen was active against the identified pathogen(s) based on in vitro susceptibility testing and administered within 12 hours following blood culture collection. Appropriate antimicrobial treatment also had to be prescribed for at least 24 hours. However, the total duration of antimicrobial therapy was at the discretion of the treating physicians. The Charlson comorbidity score was calculated using ICD-9-CM codes abstracted from the index hospitalization employing MS-DRG Grouper version 26.

\section{Antimicrobial Monitoring}

From January 2002 through the present, Barnes-Jewish Hospital utilized an antibiotic control program to help guide antimicrobial therapy. During this time, the use of cefepime and gentamicin was unrestricted. However, initiation of intravenous ciprofloxacin, imipenem/cilastatin, meropenem, or piperacillin/tazobactam was restricted and required preauthorization from either a clinical pharmacist or infectious diseases physician. Each intensive care unit (ICU) had a clinical pharmacist who reviewed all antibiotic orders to insure that dosing and interval of antibiotic administration was adequate for individual patients based on body size, renal function, and the resuscitation status of the patient. After daytime hours, the on-call clinical pharmacist reviewed and approved the antibiotic orders. The initial antibiotic dosages for the antibiotics employed for the treatment of Gram-negative infections at Barnes-Jewish Hospital were as follows: cefepime, 1 to 2 grams every eight hours; pipercillin-tazobactam, 4.5 grams every six 
hours; imipenem, 0.5 grams every six hours; meropenem, 1 gram every eight hours; ciprofloxacin, $400 \mathrm{mg}$ every eight hours; gentamicin, $5 \mathrm{mg} / \mathrm{kg}$ once daily.

Starting in June 2005, a sepsis order set was implemented in the emergency department, general medical wards, and the intensive care units with the intent of standardizing empiric antibiotic selection for patients with sepsis based on the infection type (ie, community-acquired pneumonia, healthcare-associated pneumonia, intra-abdominal infection, etc) and the hospital's antibiogram. ${ }^{14,15}$ However, antimicrobial selection, dosing, and de-escalation of therapy were still optimized by clinical pharmacists in these clinical areas.

\section{Antimicrobial Susceptibility Testing}

The microbiology laboratory performed antimicrobial susceptibility testing of the Gram-negative blood isolates using the disk diffusion method according to guidelines and breakpoints established by the Clinical Laboratory and Standards Institute (CLSI) and published during the inclusive years of the study. ${ }^{16,17}$ Zone diameters obtained by disk diffusion testing were converted to minimum inhibitory concentrations (MICs in $\mathrm{mg} / \mathrm{L}$ ) by linear regression analysis for each antimicrobial agent using the BIOMIC® $\mathrm{V}^{3}$ antimicrobial susceptibility system (Giles Scientific, Inc., Santa Barbara, CA). Linear regression algorithms contained in the software of this system were determined by comparative studies correlating microbroth dilution-determined MIC values with zone sizes obtained by disk diffusion testing. ${ }^{18}$

\section{Data Analysis}

Continuous variables were reported as mean \pm the standard deviation, or median and quartiles. The Student's $t$ test was used when comparing normally distributed data, and the Mann-Whitney $U$ test was employed to analyze nonnormally distributed data. Categorical data were expressed as frequency distributions and the Chi-squared test was used to determine if differences existed between groups. We performed multiple logistic regression analysis to identify clinical risk factors that were associated with hospital mortality (SPSS, Inc., Chicago, IL). All risk factors from Table 1, as well as the individual pathogens examined, were included in the corresponding multivariable analysis with the exception of acquired organ dysfunction (considered a secondary outcome). All tests were two-tailed, and a $P$ value $<0.05$ was determined to represent statistical significance.

\section{RESULTS}

\section{Patient Characteristics}

Included in the study were 535 consecutive patients with severe sepsis attributed to Pseudomonas aeruginosa, Acinetobacter species, or Enterobacteriaceae bacteremia, of whom $233(43.6 \%)$ died during their hospitalization.

\begin{tabular}{|c|c|c|c|}
\hline Variable & $\begin{array}{l}\text { Hospital } \\
\text { Survivors } \\
(\mathrm{n}=302)\end{array}$ & $\begin{array}{l}\text { Hospital } \\
\text { Nonsurvivors } \\
(\mathrm{n}=233)\end{array}$ & $P$ value \\
\hline Age, years & $57.9 \pm 16.2$ & $60.3 \pm 15.8$ & 0.091 \\
\hline Male & $156(51.7)$ & $132(56.7)$ & 0.250 \\
\hline \multicolumn{4}{|l|}{ Infection onset source } \\
\hline Community-acquired & $31(10.3)$ & $15(6.4)$ & 0.005 \\
\hline Healthcare-associated community-onset & $119(39.4)$ & $68(29.2)$ & \\
\hline Healthcare-associated hospital-onset & $152(50.3)$ & $150(64.4)$ & \\
\hline \multicolumn{4}{|l|}{ Underlying co-morbidities } \\
\hline CHF & $43(14.2)$ & $53(22.7)$ & 0.011 \\
\hline COPD & $42(13.9)$ & $56(24.0)$ & 0.003 \\
\hline Chronic kidney disease & $31(10.3)$ & $41(17.6)$ & 0.014 \\
\hline Liver disease & $34(11.3)$ & $31(13.3)$ & 0.473 \\
\hline Active malignancy & $100(33.1)$ & $83(35.6)$ & 0.544 \\
\hline Diabetes & $68(22.5)$ & $50(21.5)$ & 0.770 \\
\hline Charlson co-morbidity score & $4.5 \pm 3.5$ & $5.2 \pm 3.9$ & 0.041 \\
\hline APACHE $\|$ score & $21.8 \pm 6.1$ & $27.1 \pm 6.2$ & $<0.001$ \\
\hline ICU admission & $221(73.2)$ & $216(92.7)$ & $<0.001$ \\
\hline Vasopressors & $137(45.4)$ & $197(84.5)$ & $<0.001$ \\
\hline Mechanical ventilation & $124(41.1)$ & $183(78.5)$ & $<0.001$ \\
\hline Drotrecogin alfa (activated) & $6(2.0)$ & $21(9.0)$ & $<0.001$ \\
\hline \multicolumn{4}{|l|}{ Dysfunctional acquired organ systems } \\
\hline Cardiovascular & $149(49.3)$ & $204(87.6)$ & $<0.001$ \\
\hline Respiratory & $141(46.7)$ & $202(86.7)$ & $<0.001$ \\
\hline Renal & $145(48.0)$ & $136(58.4)$ & 0.017 \\
\hline Hepatic & $13(4.3)$ & $27(11.6)$ & 0.001 \\
\hline Hematologic & $103(34.1)$ & $63(27.0)$ & 0.080 \\
\hline Neurologic & $11(3.6)$ & $19(8.2)$ & 0.024 \\
\hline$\geq 2$ Dysfunctional acquired organ systems & $164(54.3)$ & $213(91.4)$ & $<0.001$ \\
\hline \multicolumn{4}{|l|}{ Source of bloodstream infection } \\
\hline Lungs & $95(31.5)$ & $127(54.5)$ & $<0.001$ \\
\hline Urinary tract & $92(30.5)$ & $45(19.3)$ & \\
\hline Central venous catheter & $30(9.9)$ & $16(6.9)$ & \\
\hline Intra-abdominal & $63(20.9)$ & $33(14.2)$ & \\
\hline Unknown & $22(7.3)$ & $12(5.2)$ & \\
\hline Prior antibiotics* & $103(34.1)$ & $110(47.2)$ & 0.002 \\
\hline
\end{tabular}

NOTE: Values indicate $\mathrm{N}(\%)$ and meant SD.

Abbreviations: APACHE, Acute Physiologic and Chronic Health Evaluation; CHF, congestive heart failure; COPD, chronic obstructive pulmonary disease; ICU, intensive care unit. " In the preceding 30 days.

The mean age was $58.9 \pm 16.0$ years (range, 18 to 96 years) with $288(53.8 \%)$ males and $247(46.2 \%)$ females. The infection sources included community-acquired $(\mathrm{n}=$ $46,8.6 \%)$, healthcare-associated community-onset $(\mathrm{n}=$ $187,35.0 \%$ ), and healthcare-associated hospital-onset (n $=302,56.4 \%)$. Hospital nonsurvivors were statistically more likely to have a healthcare-associated hospital-onset infection, congestive heart failure, chronic obstructive pulmonary disease, chronic kidney disease, ICU admission, need for mechanical ventilation and/or vasopressors, administration of drotrecogin alfa (activated), prior antibiotic administration, the lungs as the source of infection, acquired dysfunction of the cardiovascular, respiratory, renal, hepatic, and neurologic organ systems, and greater APACHE II and Charlson co-morbidity scores compared to hospital survivors (Table 1). Hospital nonsurvivors were also statistically less likely to have a healthcare-associated community-onset infection and a urinary source of infection compared to hospital survivors (Table 1). 


\begin{tabular}{|c|c|c|c|c|c|}
\hline Bacteria & $\begin{array}{l}\text { Hospital } \\
\text { Survivors } \\
(n=302)\end{array}$ & $\begin{array}{l}\text { Hospital } \\
\text { Nonsurvivors } \\
(n=233)\end{array}$ & $P$ value & $\begin{array}{l}\text { Percent } \\
\text { Resistant }^{\dagger}\end{array}$ & $\begin{array}{l}\text { Pathogen- } \\
\text { Specific } \\
\text { Mortality } \\
\text { Rate }\end{array}$ \\
\hline Enterobacteriaceae & $241(79.8)$ & $143(61.4)$ & $<0.001$ & 9.1 & 37.2 \\
\hline Pseudomonas aeruginosa & $47(15.6)$ & $67(28.8)$ & $<0.001$ & 16.7 & 58.8 \\
\hline Acinetobacter species & $22(7.3)$ & $27(11.6)$ & 0.087 & 71.4 & 55.1 \\
\hline
\end{tabular}

NOTE: Values indicate N (\%). * For the comparison of hospital survivors to nonsurvivors. ${ }^{\dagger}$ To the initially prescribed antibiotic regimen.

\section{Microbiology}

Among the 547 Gram-negative bacteria isolated from blood, the most common were Enterobacteriaceae (Escherichia coli, Klebsiella species, Enterobacter species) $(70.2 \%)$ followed by Pseudomonas aeruginosa $(20.8 \%)$ and Acinetobacter species $(9.0 \%)$ (Table 2). Nine patients had two different Enterobacteriaceae species isolated from their blood cultures, and three patients had an Enterobacteriaceae species and Pseudomonas aeruginosa isolated from their blood cultures. Hospital nonsurvivors were statistically more likely to be infected with Pseudomonas aeruginosa and less likely to be infected with Enterobacteriaceae. The pathogen-specific hospital mortality rate was significantly greater for Pseudomonas aeruginosa and Acinetobacter species compared to Enterobacteriaceae $(P<$ 0.001 and $P=0.008$, respectively).

\section{Antimicrobial Treatment and Resistance}

Among the study patients, $358(66.9 \%)$ received cefepime, $102(19.1 \%)$ received piperacillin-tazobactam, and $75(14.0 \%)$ received a carbapenem (meropenem or imipenem) as their initial antibiotic treatment. There were $169(31.6 \%)$ patients who received initial combination therapy with either an aminoglycoside (n $=99,58.6 \%)$ or ciprofloxacin ( $\mathrm{n}=70,41.4 \%)$. Eighty-two $(15.3 \%)$ patients were infected with a pathogen that was resistant to the initial antibiotic treatment regimen [cefepime $(\mathrm{n}=41 ; 50.0 \%)$, piperacillin-tazobactam $(\mathrm{n}=25 ; 30.5 \%)$, or imipenem/meropenem $(\mathrm{n}=16 ; 19.5 \%)$, plus either an aminoglycoside or ciprofloxacin $(\mathrm{n}=28 ; 34.1 \%)]$, and were classified as receiving inappropriate initial antibiotic therapy. Among the $453(84.7 \%)$ patients infected with a pathogen that was susceptible to the initial antibiotic regimen, there was no relationship identified between minimum inhibitory concentration values and hospital mortality.

Patients infected with a pathogen resistant to the initial antibiotic regimen had significantly greater risk of hospital mortality $(63.4 \%$ vs $40.0 \% ; P<0.001)$ (Figure 1 ). For the 82 individuals infected with a pathogen that was resistant to the initial antibiotic regimen, no difference in hospital mortality was observed among those prescribed initial combination treatment with an aminoglycoside

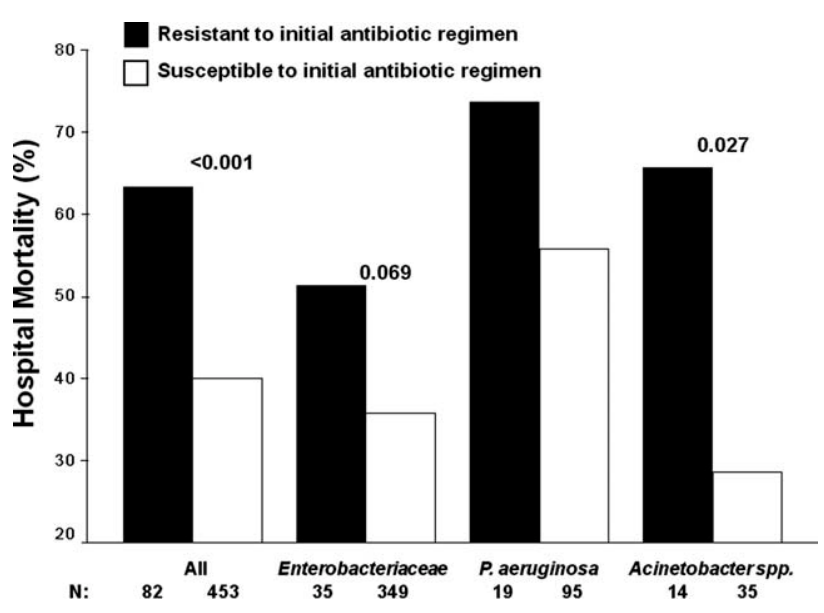

FIG. 1. Hospital mortality according to whether the isolated Gram-negative bacterial isolate was susceptible or resistant to the initially prescribed antibiotic regimen.

$(\mathrm{n}=17)(64.7 \%$ vs $61.1 \% ; P=0.790)$ or ciprofloxacin $(\mathrm{n}=11)(72.7 \%$ vs $61.1 \% ; P=0.733)$ compared to monotherapy $(\mathrm{n}=54)$. Similarly, among the patients infected with a pathogen that was susceptible to the initial antibiotic regimen, there was no difference in hospital mortality among those whose bloodstream isolate was only susceptible to the prescribed aminoglycoside $(\mathrm{n}=12)$ compared to patients with isolates that were susceptible to the prescribed beta-lactam antibiotic $(\mathrm{n}=441)(41.7 \%$ vs $39.9 \% ; P=0.902)$.

Logistic regression analysis identified infection with a pathogen resistant to the initial antibiotic regimen [adjusted odds ratio (AOR), 2.28; 95\% confidence interval (CI), 1.69-3.08; $P=0.006]$, increasing APACHE II scores (1-point increments) (AOR, 1.13; $95 \%$ CI, $1.10-1.15 ; P<0.001)$, the need for vasopressors (AOR, 2.57; 95\% CI, 2.15-3.53; $P<0.001$ ), the need for mechanical ventilation (AOR, 2.54; 95\% CI, 2.19-3.47; $P<0.001$ ), healthcare-associated hospital-onset infection (AOR, 1.67; 95\% CI, 1.32-2.10; $P=0.027)$, and infection with Pseudomonas aeruginosa (AOR, 2.21; 95\% CI, 1.74-2.86; $P=0.002$ ) as independent risk factors for hospital mortality (Hosmer-Lemeshow goodness-of-fit test $=0.305)$. The model explained between $29.7 \%$ (Cox and Snell $R$ square) and $39.8 \%$ (Nagelkerke $R$ squared) of the variance in hospital mortality, and correctly classified $75.3 \%$ of cases.

\section{Secondary Outcomes}

Two or more acquired organ system derangements occurred significantly more often among patients with a pathogen resistant to the initial antibiotic regimen compared to those infected with susceptible isolates $(84.1 \%$ vs $68.0 \% ; P=0.003)$. Hospital length of stay was significantly longer for patients infected with a pathogen resistant to the initial antibiotic regimen compared to those infected with susceptible isolates [39.9 \pm 50.6 days (median 27 days; quartiles 12 days 
and 45.5 days) vs $21.6 \pm 22.0$ days (median 15 days; quartiles 7 days and 30 days); $P<0.001]$.

\section{DISCUSSION}

Our study demonstrated that hospital nonsurvivors with severe sepsis attributed to Gram-negative bacteremia had significantly greater rates of resistance to their initially prescribed antibiotic regimen compared to hospital survivors. This observation was confirmed in a multivariate analysis controlling for severity of illness and other potential confounding variables. Additionally, acquired organ system derangements and hospital length of stay were greater for patients infected with Gram-negative pathogens resistant to the empiric antibiotic regimen. We also observed no survival advantage with the use of combination antimicrobial therapy for the subgroup of patients whose pathogens were resistant to the initially prescribed antibiotic regimen. Lastly, no difference in mortality was observed for patients with bacterial isolates that were susceptible only to the prescribed aminoglycoside compared to those with isolates susceptible to the prescribed beta-lactam antibiotic.

Several previous investigators have linked antibiotic resistance and outcome in patients with serious infections attributed to Gram-negative bacteria. Tam et al. examined 34 patients with Pseudomonas aeruginosa bacteremia having elevated MICs to piperacillin-tazobactam $(\geq 32 \mu \mathrm{g} / \mathrm{mL})$ that were reported as susceptible. ${ }^{19}$ In seven of these cases, piperacillin-tazobactam was prescribed empirically, whereas other agents directed against Gram-negative bacteria were employed in the other patients (carbapenems, aminoglycosides). Thirty-day mortality was significantly greater for the patients treated with piperacillin-tazobactam $(85.7 \%$ vs $22.2 \%$; $P=0.004)$, and a multivariate analysis found treatment with piperacillintazobactam to be independently associated with 30day mortality. Similarly, Bhat et al. examined 204 episodes of bacteremia caused by Gram-negative bacteria for which patients received cefepime. ${ }^{20}$ Patients infected with a Gram-negative bacteria having an MIC to cefepime greater than, or equal to, $8 \mu \mathrm{g} / \mathrm{mL}$ had a significantly greater 28 -day mortality compared to patients infected with isolates having an MIC to cefepime that was less than $8 \mu \mathrm{g} / \mathrm{mL} \quad(54.8 \%$ vs $24.1 \% ; P=0.001)$.

Our findings are consistent with earlier studies of patients with serious Gram-negative infections including bacteremia and nosocomial pneumonia. Micek et al. showed that patients with Pseudomonas aeruginosa bacteremia who received inappropriate initial antimicrobial therapy had a greater risk of hospital mortality compared to patients initially treated with an antimicrobial regimen having activity for the Pseudomonas isolate based on in vitro susceptibility testing. ${ }^{21}$ Similarly, Trouillet et al., ${ }^{22}$ Beardsley et al., ${ }^{23}$ and Heyland et al. ${ }^{24}$ found that combination antimicrobial regimens directed against Gram-negative bacteria in patients with nosocomial pneumonia were more likely to be appropriate based on the antimicrobial susceptibility patterns of the organisms compared to monotherapy. In a more recent study, Micek et al. demonstrated that combination antimicrobial therapy directed against severe sepsis attributed to Gram-negative bacteria was associated with improved outcomes compared to monotherapy, especially when the combination agent was an aminoglycoside. ${ }^{25}$ However, empiric combination therapy that included an aminoglycoside was also associated with increased nephrotoxicity which makes the empiric use of aminoglycosides in all patients with suspected Gramnegative severe sepsis problematic. ${ }^{25,26}$ Nevertheless, the use of combination therapy represents a potential strategy to maximize the administration of appropriate treatment for serious Gram-negative bacterial infections.

Rapid assessment of antimicrobial susceptibility is another strategy that offers the possibility of identifying the resistance pattern of Gram-negative pathogens quickly in order to provide more appropriate treatment. Bouza et al. found that use of a rapid E-test on the respiratory specimens of patients with ventilatorassociated pneumonia was associated with fewer days of fever, fewer days of antibiotic administration until resolution of the episode of ventilator-associated pneumonia, decreased antibiotic consumption, less Clostridium difficile-associated diarrhea, lower costs of antimicrobial agents, and fewer days receiving mechanical ventilation. ${ }^{27}$ Other methods for the rapid identification of resistant bacteria include real-time polymerase chain reaction assays based on hybridization probes to identify specific resistance mechanisms in bacteria. ${ }^{28}$ Application of such methods for identification of broad categories of resistance mechanisms in Gram-negative bacteria offer the possibility of tailoring initial antimicrobial regimens in order to provide appropriate therapy in a more timely manner.

Our study has several important limitations that should be noted. First, the study was performed at a single center and the results may not be generalizable to other institutions. However, the findings from other investigators corroborate the importance of antimicrobial resistance as a predictor of outcome for patients with serious Gram-negative infections. ${ }^{19,20}$ Additionally, a similar association has been observed in patients with methicillin-resistant Staphylococcus aureus bacteremia, supporting the more general importance of antimicrobial resistance as an outcome predictor. ${ }^{29}$ Second, the method employed for determining MICs was a literature-based linear regression method correlating disk diffusion diameters with broth dilution MIC determinations. Therefore, the lack of correlation we observed between MIC values and outcome for susceptible Gram-negative isolates associated with severe sepsis requires further confirmation. Third, we only examined 
3 antibiotics, or antibiotic classes, so our results may not be applicable to other agents. This also applies to doripenem, as we did not have that specific carbapenem available at the time this investigation took place.

Another important limitation of our study is the relatively small number of individuals infected with a pathogen that was resistant to the initial treatment regimen, or only susceptible to the aminoglycoside when combination therapy was prescribed. This limited our ability to detect meaningful associations in these subgroups of patients, to include whether or not combination therapy influenced their clinical outcome. Finally, we did not examine the exact timing of antibiotic therapy relative to the onset of severe sepsis. Instead we used a 12-hour window from when subsequently positive blood cultures were drawn to the administration of initial antibiotic therapy. Other investigators have shown that delays in initial appropriate therapy of more than one hour for patients with septic shock increases the risk of death. ${ }^{9,30}$ Failure to include the exact timing of therapy could have resulted in a final multivariate model that includes prediction variables that would not otherwise have been incorporated.

In summary, we demonstrated that resistance to the initial antibiotic treatment regimen was associated with a greater risk of hospital mortality in patients with severe sepsis attributed to Gram-negative bacteremia. These findings imply that more rapid assessment of antimicrobial susceptibility could result in improved prescription of antibiotics in order to maximize initial administration of appropriate therapy. Future studies are required to address whether rapid determination of antimicrobial susceptibility can result in more effective administration of appropriate therapy, and if this can result in improved patient outcomes.

Disclosure: Dr. Kollef's effort was supported in part by the Barnes-Jewish Hospital Foundation. This project was supported in part by an unrestricted grant from Johnson \& Johnson. All other authors had no funding and no conflicts of interest to report.

\section{References}

1. Kollef MH, Sherman G, Ward S, Fraser VJ. Inadequate antimicrobial treatment of infections: a risk factor for hospital mortality among critically ill patients. Chest. 1999;115:462-474.

2. Dhainaut JF, Laterre PF, LaRosa SP, et al. The clinical evaluation committee in a large multicenter phase 3 trial of drotrecogin alfa (activated) in patients with severe sepsis (PROWESS): role, methodology, and results. Crit Care Med. 2003;31:2291-2301.

3. Garnacho-Montero J, Garcia-Garmendia JL, Barrero-Almodovar A, Jimenez-Jimenez FJ, Perez-Paredes C, Ortiz-Leyba C. Impact of adequate empical antibiotic therapy on the outcome of patients admitted to the intensive care unit with sepsis. Crit Care Med. 2003; 31:2742-2751.

4. Harbarth S, Garbino J, Pugin J, Romand JA, Lew D, Pittet D. Inappropriate initial antimicrobial therapy and its effect on survival in a clinical trial of immunomodulating therapy for severe sepsis. Am J Med. 2003;115:529-535.

5. Arias CA, Murray BE. Antibiotic-resistant bugs in the 21st centurya clinical super-challenge. N Engl J Med. 2009;360:439-443.

6. Boucher H, Talbot GH, Bradley JS, et al. Bad bugs, no drugs: no ESKAPE! An update from the Infectious Diseases Society of America. Clin Infect Dis. 2009;48:1-12.
7. Kollef MH. Broad-spectrum antimicrobials and the treatment of serious bacterial infections: getting it right up front. Clin Infect Dis. 2008;47:S3-S13.

8. Barochia AV, Cui X, Vitberg D, et al. Bundled care for septic shock: an analysis of clinical trials. Crit Care Med. 2010;38:668-678.

9. Ferrer R, Artigas A, Suarez D, et al. Effectiveness of treatments for severe sepsis: a prospective, multicenter, observational study. Am J Respir Crit Care Med. 2009;180:861-866.

10. Dellinger RP, Levy MM, Carlet JM, et al. Surviving Sepsis Campaign: international guidelines for management of severe sepsis and septic shock: 2008. Crit Care Med. 2008;36:296-327.

11. Knaus WA, Draper EA, Wagner DP, Zimmerman JE. APACHE II: a severity of disease classification system. Crit Care Med. 1985;13: 818-829.

12. Klevens RM, Morrison MA, Nadle J, et al. Invasive methicillin-resistant Staphylococcus aureus infections in the United States. JAMA. 2007;298:1763-1771.

13. Angus DC, Linde-Zwirble WT, Lidicker J, Clermont G, Carcillo J, Pinsky MR. Epidemiology of severe sepsis in the United States: analysis of incidence, outcome, and associated costs of care. Crit Care Med. 2001;29:1303-1310.

14. Thiel SW, Asghar MF, Micek ST, Reichley RM, Doherty JA, Kollef MH. Hospital-wide impact of a standardized order set for the management of bacteremic severe sepsis. Crit Care Med. 2009;37: 819-824.

15. Micek ST, Roubinian N, Heuring T, et al. Before-after study of a standardized hospital order set for the management of septic shock. Crit Care Med. 2007;34:2707-2713.

16. National Committee for Clinical Laboratory Standards. Performance Standards for Antimicrobial Susceptibility Testing: Twelfth Informational Supplement. M100-S12. Wayne, PA: National Committee for Clinical Laboratory Standards; 2002.

17. Clinical Laboratory Standards Institute. Performance Standards for Antimicrobial Susceptibility Testing: Seventeenth Informational Supplement. M100-S17. Wayne, PA: Clinical Laboratory Standards Institute; 2007.

18. D'Amato RF, Hochstein L, Vernaleo JR, et al. Evaluation of the BIOGRAM antimicrobial susceptibility test system. J Clin Microbiol. 1985;22:793-798.

19. Tam VH, Gamez EA, Weston JS, et al. Outcomes of bacteremia due to Pseudomonas aeruginosa with reduced susceptibility to piperacillin-tazobactam: implications on the appropriateness of the resistance breakpoint. Clin Infect Dis. 2008;46:862-867.

20. Bhat SV, Peleg AY, Lodise TP Jr, et al. Failure of current cefepime breakpoints to predict clinical outcomes of bacteremia caused by Gram-negative organisms. Antimicrob Agents Chemother. 2007;51: 4390-4395.

21. Micek ST, Lloyd AE, Ritchie DJ, Reichley RM, Fraser VJ, Kollef MH. Pseudomonas aeruginosa bloodstream infection: importance of appropriate initial antimicrobial treatment. Antimicrob Agents Chemother. 2005;49:1306-1311.

22. Trouillet JL, Chastre J, Vuagnat A. Ventilator-associated pneumonia caused by potentially drug-resistant bacteria. Am J Respir Crit Care Med. 1998;157:531-539.

23. Beardsley JR, Williamson JC, Johnson JW, Ohl CA, Karchmer TB, Bowton DL. Using local microbiologic data to develop institutionspecific guidelines for the treatment of hospital-acquired pneumonia. Chest. 2006;130:787-793.

24. Heyland DK, Dodek P, Muscedere J, et al. Randomized trial of combination versus monotherapy for the empiric treatment of suspected ventilator-associated pneumonia. Crit Care Med. 2008;36:737-744.

25. Micek ST, Welch EC, Khan J, et al. Empiric combination antibiotic therapy is associated with improved outcome in Gram-negative sepsis: a retrospective analysis. Antimicrob Agents Chemother. 2010;54: 1742-1748.

26. Leibovici L, Paul M, Poznanski O, et al. Monotherapy versus betalactam-aminoglycoside combination treatment for Gram-negative bacteremia: a prospective, observational study. Antimicrob Agents Chemother. 1997;41:1127-1133.

27. Bouza E, Torres MV, Radice C, et al. Direct E-test (AB Biodisk) of respiratory samples improves antimicrobial use in ventilator-associated pneumonia. Clin Infect Dis. 2007;44:382-387.

28. Oxacelay C, Ergani A, Naas T, et al. Rapid detection of CTX-M-producing Enterobacteriaceae in urine samples. J Antimicrob Chemother. 2009; 64:986-989.

29. Soriano A, Marco F, Martínez JA, et al. Influence of vancomycin minimum inhibitory concentration on the treatment of methicillin-resistant Staphylococcus aureus bacteremia. Clin Infect Dis. 2008;46: 193-200.

30. Kumar A, Roberts D, Wood KE, et al. Duration of hypotension before initiation of effective antimicrobial therapy is the critical determinant of survival in human septic shock. Crit Care Med. 2006;34: 1589-1596. 